\title{
9.
}

\section{Notiz über einen elementaren Satz der Statik.}

(Von dem Herrn Ingenieur Dr. A. Winckler zu Carlsrube in Baden.)

Die systematische Darstellung der elementaren Statik, welche die Lehrbücher auf verschiedenen Wegen anstreben, würde, wie es scheint, wesentlich gefördert werden, wenn schon gleich im Anfange das allgemeine Gesetz der virtuellen Momente eingeführt und in den einzelnen Fällen begründet würde.

Die Möglichkeit davon ist leicht zu sehen. Es ergeben sich schon beim Parallelogramm der Kräfte die Sätze über die statischen und virtuellen Momente aus den Projectionen der Kräfte auf zwei senkrechte Axen, und es folgen daraus, fast ohne Weiteres, die Sätze über die parallelen Kräfte.

Bezeichnen nämlich $\boldsymbol{P}$ und $\boldsymbol{Q}$ zwei auf einen Punct wirkende Kräfte und $\boldsymbol{R}$ ihre Resultante; legt man durch jenen Punct eine gerade Linie, welche, etwa aufserhalb des Winkels $(\boldsymbol{P Q})$ liegend, mit den Kräften $\boldsymbol{P}, \boldsymbol{Q}, \boldsymbol{R}$ resp. die Winkel $a, b, c$ macht, und projicirt die Kräfte auf diese und auf eine darauf senkrechte Gerade, so findet sich unmittelbar:

$$
\begin{aligned}
& \boldsymbol{R} \sin c=\boldsymbol{P} \sin a+\boldsymbol{Q} \sin b, \\
& \boldsymbol{R} \cos c=\boldsymbol{P} \cos a+\boldsymbol{Q} \cos b .
\end{aligned}
$$

Bezeichnet ferner $\delta$ die Entfernung eines auf jener Geraden angenommenen Punctes $\boldsymbol{C}$ vom Angriffspuncte $\boldsymbol{A}$, und setzt man

$$
\begin{aligned}
& \delta \sin a=p, \quad \delta \sin b=q, \quad \delta \sin c=r, \\
& \delta \cos a=\nu^{\prime}, \quad \delta \cos b=q^{\prime}, \quad \delta \cos c=r^{\prime},
\end{aligned}
$$

so sind $p, q, r$ die Hebels-Arme und $p^{\prime}, q^{\prime}, r^{\prime}$ die virtuellen Geschwindigkeiten der Kräfte in Bezug auf den Punct $C$, welche mit den sin. und cos. der Winkel $a, b, c$ gleiche Zeichen haben.

Dies vorausgesetzt, erhält man die Gleichungen

$$
\begin{gathered}
\boldsymbol{R} \boldsymbol{r}=\boldsymbol{P} \boldsymbol{p}+\boldsymbol{Q} \boldsymbol{q}, \\
\boldsymbol{R} \boldsymbol{r}^{\prime}=\boldsymbol{P}_{\boldsymbol{p}^{\prime}}+\boldsymbol{Q} q^{\prime}, \\
\boldsymbol{p}^{2}+\boldsymbol{p}^{\prime 2}=\boldsymbol{q}^{2}+\boldsymbol{q}^{\prime 2}=\boldsymbol{r}^{2}+\boldsymbol{r}^{\prime 2},
\end{gathered}
$$

durch welche die Resultante der Gröfse und Richtung nach bestimmt wird. 
Hieraus ergeben sich nun auch die Gleichungen für die parallelen Kräfte, wenn man den Durchschnittspunct $A$ der Richtungslinien der Kräfte auf $\boldsymbol{C A}$, von $\boldsymbol{C}$ hinweg ins Unendliche fortrücken läfst, während die Kräfte $\boldsymbol{P}, \boldsymbol{Q}$ und die Hebels-Arme unveränderlich bleiben, wogegen $p^{\prime}, q^{\prime}, r^{\prime}$, ohne Ende wachsend, stets mehr und mehr einander gleich werden.

Alsdann findet sich nemlich:

1. Wenn der Winkel $(\boldsymbol{P} \boldsymbol{Q})<90$ ist, aus

$$
\boldsymbol{R} \boldsymbol{r}^{\prime}=\boldsymbol{P}_{\boldsymbol{p}^{\prime}}+\boldsymbol{Q}_{\boldsymbol{q}^{\prime}} \text { die Gleichung } \boldsymbol{R}=\boldsymbol{P}+\boldsymbol{Q} .
$$

(Die Gleichung für die statischen Momente bleibt, wie in den folgenden Fällen, unverändert.)

2. Wenn $(\boldsymbol{P Q})>90$ und $\boldsymbol{P}>\boldsymbol{Q}$ ist, also $\boldsymbol{R}$ näher an $\boldsymbol{P}$ als an $\boldsymbol{Q}$ liegt, aus der diesem Falle entsprechenden Gleichung

$$
\boldsymbol{R} \boldsymbol{r}^{\prime}=\boldsymbol{P}_{\boldsymbol{p}^{\prime}}-\boldsymbol{Q} \boldsymbol{q}^{\prime}, \text { die folgende }: \boldsymbol{R}=\boldsymbol{P}-\boldsymbol{Q} \text {. }
$$

3. Wenn $(\boldsymbol{P Q})>90$ und $\boldsymbol{P}=\boldsymbol{Q}$ ist, also $\boldsymbol{R}$ den Winkel der beiden Seitenkräfte halbirt, aus der entsprechenden Gleichung

$$
\boldsymbol{R} \boldsymbol{r}^{\prime}=\boldsymbol{P}_{\boldsymbol{p}^{\prime}}-\boldsymbol{P} \boldsymbol{q}^{\prime} \text {, die Resultante } \boldsymbol{R}=\mathbf{0} \text {. }
$$

Der Angriffspunct dieser Mittleren, welche in einer auf der der beiden Seitenkräfte senkrechten Richtung wirkt, liegt im Unendlichen; ihr Werth ist Null und man sagt daher, zwei gleiche entgegengesetzte parallele Kräfte haben keine Mittelkraft. Ihr Moment aber behält stets den Werth $\boldsymbol{P}(\boldsymbol{p}+\boldsymbol{q})$.

Wie beim Parallelogramm, läfst sich das Verfahren auch auf beliebige Kräfte in einer Ebene, auf das Parallelepiped und auf beliebige im Raume wirkende Kräfte, allmälig fortschreitend, anwenden; was keiner weitern Ausführung bedarf.

Einen so einfachen Gegenstand berührt zu haben, möge der Umstand rechtfertigen, dafs auch Poisson in seine Mechanik (T. I. no. 43, 44, 50.) zwei verschiedene Herleitungen der obigen Sätze über die parallelen Kräfte aufgenommen hat.

Carlsruhe, den 12. November 1852. 